\title{
Current smoking is associated with extracranial carotid atherosclerotic stenosis but not with intracranial large artery disease
}

Ruijun Ji $i^{1,2,3,4,5}$, Yuesong Pan ${ }^{2,3}$, Hongyi Yan ${ }^{2,3}$, Runhua Zhang ${ }^{2,3}$, Gaifen Liu' ${ }^{1,2,3,4}$, Penglian Wang ${ }^{1,2,3,4}$, Yilong Wang 1,2,3,4, Hao Li ${ }^{2,3}$, Xingquan Zhao ${ }^{1,2,3,4}$, Yongjun Wang ${ }^{1,2,3,4,6^{*}}$ On behalf of The Chinese Intracranial Atherosclerosis (CICAS) Study investigators

\begin{abstract}
Background: Accumulating evidence has shown that cigarette smoking is an important risk factor for ischemic stroke. However, it is not clear about the potential mechanisms through which cigarette smoking affects stroke risk. In the study, we aimed to investigate the relationship between cigarette smoking and the occurrence of extracranial (ECAS) and intracranial atherosclerotic stenosis (ICAS).

Methods: We analyzed patients enrolled in the Chinese intracranial atherosclerosis (CICAS), which was a prospective, multicenter, hospital-based cohort study. Smoking status was classified into never, former and current smoking. For those patients with current smoking, data on time duration (year) and extent (the number of cigarette smoked per day) was recorded and pack year of smoking was calculated. ICAS was evaluated with 3-dimentional time-of-flight MRA and ECAS was evaluated with cervical ultrasonography or contrast-enhanced MRA. Multivariable Logistic regression was performed to identify the association between smoking status and the occurrence of ECAS and ICAS.

Results: A total of 2656 patients (92.7\%) of acute ischemic stroke and 208 (7.3\%) of transient ischemic attack were analyzed. The mean age was $61.9 \pm 11.2$ and 67.8\% were male. There were 141 (4.9\%) patients had only ECAS, 1074 (37.5\%) had only ICAS, and 261 (9.1\%) had both ECAS and ICAS. Current smoking was significantly associated with the occurrence of ECAS (adjusted $\mathrm{OR}=1.47,95 \% \mathrm{Cl}=1.09-1.99, P<0.01$ ). In addition, with 1 year of smoking increment, the risk of ECAS increased by $1.1 \%$ (adjusted $\mathrm{OR}=1.011 ; 95 \% \mathrm{Cl}=1.003-1.019 ; P=0.005$ ); with one cigarette smoked per day increment, the risk of ECAS increased by 1.0\% (adjusted $\mathrm{OR}=1.010 ; 95 \% \mathrm{Cl}=1.001-1.020 ; P=0.03$ ); and with one pack year of smoking increment, the risk of ECAS increased by $0.7 \%$ (adjusted $\mathrm{OR}=1.007 ; 95 \% \mathrm{Cl}=1.002-1.012 ; P<0.01$ ). However, no significant association was found between smoking status and the occurrence of ICAS.
\end{abstract}

Conclusion: A dose-response relationship was identified between cigarette smoking and the occurrence of ECAS, but not ICAS. Further studies on molecular mechanisms were warranted.

Keywords: Stroke, Smoking, Extracranial atherosclerotic stenosis, Intracranial atherosclerotic stenosis, Association

\footnotetext{
* Correspondence: yongjunwang1962@gmail.com

'Department of Neurology, Tiantan Comprehensive Stroke Center, Tiantan Hospital, Capital Medical University, Beijing, China

${ }^{2}$ China National Clinical Research Center for Neurological Diseases, Beijing, China

Full list of author information is available at the end of the article
} 


\section{Background}

Cigarette smoking is responsible for approximately five million deaths every year and there are still an estimated 1.1 billion smokers worldwide [1-3]. Accumulating evidence has shown that cigarette smoking is an important risk factor for first ischemic stroke [4]. In contrast to extensive data on association between cigarette smoking and risk for first stroke, data on its association with recurrent stroke are sparse [5]. In the Cardiovascular Health Study, cigarette smoking was associated with a 2 -fold increased risk for stroke recurrence in the elderly [6].

The mechanisms through which cigarette smoking affects stroke risk have been a topic of interest over the last several years. Although it is not fully understood, cigarette smoking likely contributes to increased stroke risk through both short-term effect on thrombus generation in atherosclerotic arteries and long-term effect related to the development of atherosclerotic stenosis [7]. Clinically, atherosclerotic stenosis of cervicocephalic arteries could be classified into extracranial (ECAS) and intracranial atherosclerotic stenosis (ICAS). ECAS is the most common vascular lesion found in stroke patients of White. In contrast, ICAS is found more common among stroke patients of Asian, Black, and Hispanic ancestry [8]. Thus far, limited is known about the potential role of cigarette smoking in the development of ECAS and ICAS.

In the present study, we aimed to clarify the relationship between time duration, the number of cigarette smoked per day and pack years of smoking and the occurrence of ECAS and ICAS.

\section{Methods}

\section{Study population}

This study was based on the Chinese intracranial atherosclerosis (CICAS) study [9], which was a prospective, multicenter, hospital-based cohort study aimed at clarifying the prevalence and risk of recurrent stroke in patients with intracranial large artery occlusive disease. Briefly, 22 general hospitals covering a wide geographic area in China participated in the CICAS. The inclusion criteria of CICAS study were: (1) age between 18 and 80 years; (2) hospitalized with a primary diagnosis of acute ischemic stroke (AIS) or transient ischemic attack (TIA) according to the World Health Organization criteria; (3) stroke confirmed by head computerized tomography $(\mathrm{CT})$ and/or brain magnetic resonance imaging (MRI); (4) time from symptom onset to hospitalization $<7$ days. The CICAS study excluded those patients who were: (1) with known source for cardioembolism; (2) disabled before admission (modified Rankin Scale [mRS] score > 2); (3) physically or subjectively unable to comply with MRI examination; (4) clinically unstable or required close monitoring or were moribund. The CICAS study had been approved by the institutional review board (IRB) at each participating hospital. All participants provided written informed consent.

\section{Study variables \\ Clinical characteristics}

In the CICAS network, a standardized case report form (CRF) was used for data collection. For the present study, the following candidate variables were analyzed: (1) demographics (age and gender); (2) stroke risk factors: hypertension (defined by a history of hypertension, or being treated with an antihypertensive agent before admission, or diagnosed at discharge), diabetes mellitus (defined by a history of diabetes mellitus, or being treated for diabetes mellitus or glycosylated hemoglobin $\geq 7 \%$, or diagnosis at discharge), dyslipidemia (defined as a selfreported history, current use of lipid-lowering medications, total cholesterol level $\geq 5.7 \mathrm{mmol} / \mathrm{L}$, or triglyceride level $\geq 1.7 \mathrm{mmol} / \mathrm{L}$ on admission), family history of stroke, history of cerebral ischemia (including a history of ischemic stroke and TIA), history of hemorrhagic stroke (including intracerebral hemorrhage and subarachnoid hemorrhage), heart disease (defined as a history of myocardial infarction, angina pectoris, and congestive heart failure), smoking status, and heavy drinking (drinking $>2$ units per day on average for men or $>1$ unit per day on average for women); (3) comorbidities: chronic obstructive pulmonary disease (COPD), hepatic cirrhosis, peptic ulcer or previous gastrointestinal bleeding (GIB), arthritis, Alzheimer's disease/dementia, cancer and peripheral angiopathy; (4) Admission systolic (SBP) and diastolic blood pressure (DBP) ( $\mathrm{mmHg}$ ); (5) Admission National Institutes of Health Stroke Scale (NIHSS) score; (6) admission blood tests: fasting blood glucose $(\mathrm{mmol} / \mathrm{L})$, triglyceride (TG) $(\mathrm{mmol} / \mathrm{L})$, cholesterol (TC) $(\mathrm{mmol} / \mathrm{L})$, high density lipoprotein $(\mathrm{HDL})(\mathrm{mmol} / \mathrm{L})$ and low density lipoprotein (LDL) ( $\mathrm{mmol} / \mathrm{L})$.

\section{Smoking status}

In the CICAS study, smoking status was classified into never, former and current smoking. Current smoking was defined as a patient who had smoked continuously for 6 months with at least one cigarette per day. Former smoking was defined as a patient who was used to smoke but not satisfied the criteria of current smoking. For those patients with current smoking, data on time duration (year) and extent (the number of cigarette smoked per day) was recorded and pack year of smoking was calculated. The pack year is a unit for measuring the amount a person has smoked over a long period of time. It is calculated by multiplying the number of packs of cigarettes smoked per day by the number of years the person has smoked. 


\section{Vascular imaging of ECAS and ICAS}

In the CICAS study, all patients underwent conventional MRI on a 3.0-T or 1.5-T MR scanner, including sequences of 3-D time-of-flight MRA, $\mathrm{T}_{2} / \mathrm{T}_{1}$-weighted imaging, fluid-attenuated inversion recovery sequences (FLAIR), and diffusion-weighted imaging (DWI). All MRI and MRA images were read centrally by two readers who were blinded to patients' clinical information. Disagreement involving more than 10\% degree of stenosis was resolved by an appointed senior reader who decided the final classification. The following arterial segments were assessed: 1) bilateral extracranial and intracranial internal carotid artery (ICA), anterior cerebral artery (ACA) A1/A2, middle cerebral artery (MCA) M1/M2, posterior cerebral artery (PCA) P1/P2 and basilar artery (BA). The degree of intracranial stenosis on MRA was calculated using the method of the WASID study [10] and was classified into four groups: $<50 \%$ or no stenosis, $50 \%$ to $69 \%, 70 \%$ to $99 \%$, and occlusion. The extracranial internal carotid artery was evaluated with ultrasonography according to the published diagnostic criteria [11] or by contrast-enhanced MRA. In the present study, significant stenosis was defined as a more than $50 \%$ atherosclerotic stenosis or occlusion of the large intracranial and/or extracranial arteries. Based on the presence and location of cerebral atherosclerotic stenosis, patients were classified into four groups: 1) without ECAS or ICAS; 2 ) with only ECAS; 3 ) with only ICAS; and 4) with both ECAS and ICAS.

\section{Statistical analysis}

Continuous variables were summarized with mean and standard deviation (SD) or median and interquartile range (IQR); categorical variables were summarized as proportions. In univariate analysis, Chi-square or Fisher exact test was used to compare categorical variables and one way analysis of variance or Kruskal-Wallis test was employed to compare continuous variables. In multivariable analysis, Logistic regression was performed to assess association between smoking status (never, former and current smoking) and the occurrence of ECAS, ICAS and specific location of ICAS (intracranial ICA, MCA, ACA, PCA and BA). Logistic regression adjusted for demographics (age and gender), stroke risk factors, comorbidities, admission NIHSS score, admission SBP and DBP, and admission blood tests. All tests were 2-tailed and statistical significance was determined at $\alpha$ level of 0.05 . Statistical analysis was performed using SAS 9.1 (SAS Institute, Cary, NC) and SPSS 17.0 (SPSS Inc., Chicago, IL).

\section{Results}

Patient characteristics

Patient characteristics are shown in Table 1. A total of 2864 patients (2656 [92.7\%] of AIS and 208 [7.3\%] of
TIA) were enrolled in the CICAS network. The mean age was $61.9 \pm 11.2$ and $67.8 \%$ were male. There were 1568 (54.8\%) patients who never smoked, 247 (8.6\%) with former smoking and 1049 (36.6\%) with current smoking. A total number of 1476 (51.5\%) patients had either ECAS or ICAS. Compared with patients without ECAS or ICAS, those developed ECAS or ICAS were more common for older age, male gender, presence of diabetes mellitus, hypertension, dyslipidemia and cerebral ischemia, family history of stroke, current or former smoking, higher NIHSS score, higher blood glucose, and lower blood HDL (Table 1).

\section{Proportion of ECAS and ICAS}

The proportions of ECAS and ICAS in the CICAS study are shown in Additional file 1: Table S1. A total number of 141 (4.9\%) patients had only ECAS, 1074 (37.5\%) had only ICAS, and 261 (9.1\%) had both ECAS and ICAS. For those patients developing ICAS, the most frequent location was MCA stenosis (29.6\%), then followed by PCA stenosis (18.6\%), ACA stenosis (7.7\%), BA stenosis (6.3\%), and intracranial ICA (2.3\%) (Additional file 1: Table S1).

\section{Association between smoking status and ECAS or ICAS}

Association between smoking status and the occurrence of ECAS or ICAS is shown in Table 2. Compared with patients never smoked, those with current smoking were significantly associated with the occurrence of ECAS (adjusted OR $=1.47,95 \% \mathrm{CI}=1.09-1.99, P<0.01$ ). Although there is a trend that former smoking was associated with ECAS (adjusted for age, gender, and stroke risk factors), it did not reach statistical significance after adjusting all potential confounders (adjusted $\mathrm{OR}=1.42$, $95 \% \mathrm{CI}=0.92-2.18, P=0.11)$. When combining former and current smoking together, ever smoking was significantly associated with the occurrence of ECAS (adjusted $\mathrm{OR}=1.46,95 \% \mathrm{CI}=1.09-1.94, P<0.01)$. However, no similar association was found between cigarette smoking and the occurrence of ICAS or specific location of ICAS (intracranial ICA, MCA, ACA, PCA and BA) (Table 2).

\section{Association between time duration of smoking and ECAS or ICAS}

Association between time duration of smoking and the occurrence of ECAS or ICAS was investigated in the subgroup of patients who were with never and current smoking $(n=2617)$. The risk of ECAS increased steadily with longer time duration of smoking (Fig. 1a). After adjusting for all potential confounders, significant association was found between time duration of smoking and the occurrence of ECAS. With 1 year of smoking increment, the risk of developing ECAS increased by $1.1 \%$ (adjusted $\mathrm{OR}=1.011 ; 95 \% \mathrm{CI}=1.003-1.019 ; P=0.005)$. 
Table 1 Baseline characteristics

\begin{tabular}{|c|c|c|c|c|c|c|}
\hline Variables & $\begin{array}{l}\text { Overall } \\
(n=2864)\end{array}$ & $\begin{array}{l}\text { No ECAS or ICAS } \\
(n=1388)\end{array}$ & $\begin{array}{l}\text { Only ECAS } \\
(n=141)\end{array}$ & $\begin{array}{l}\text { Only ICAS } \\
(n=1074)\end{array}$ & $\begin{array}{l}\text { ECAS + ICAS } \\
(n=261)\end{array}$ & $P$ value \\
\hline Age (Mean \pm SD) & $61.9 \pm 11.2$ & $61.2 \pm 11.2$ & $64.7 \pm 10.4$ & $62.1 \pm 11.5$ & $63.6 \pm 10.1$ & $<0.001$ \\
\hline Gender (male) & 1944(67.8) & $938(67.6)$ & 110(78.0) & $696(64.8)$ & 200(76.6) & $<0.001$ \\
\hline \multicolumn{7}{|l|}{ Stroke risk factors } \\
\hline Diabetes mellitus & $991(34.6)$ & $414(29.3)$ & $50(35.5)$ & 418(38.9) & 109(41.8) & $<0.001$ \\
\hline Hypertension & 2238(78.1) & $1056(76.1)$ & $105(74.5)$ & $866(80.6)$ & $211(80.8)$ & 0.02 \\
\hline Hyperlipidemia & 2173(75.9) & 1042(75.1) & 119(84.4) & $807(75.1)$ & 205(78.5) & 0.05 \\
\hline Family history of stroke & 296(10.3) & $122(8.8)$ & 16(11.4) & $112(10.4)$ & $46(17.6)$ & $<0.001$ \\
\hline History of cerebral ischemia & 2018(70.5) & $930(67.0)$ & 117(83.0) & $772(71.9)$ & 199(76.3) & $<0.001$ \\
\hline History of hemorrhagic stroke & $54(1.9)$ & $29(2.1)$ & $3(2.1)$ & $19(1.8)$ & $3(1.2)$ & 0.73 \\
\hline Coronary heart disease & $228(8.0)$ & $102(7.4)$ & $17(12.1)$ & $88(8.2)$ & $21(8.1)$ & 0.30 \\
\hline Heavy drinking & $142(5.0)$ & $66(4.8)$ & $9(6.4)$ & $51(4.8)$ & $16(6.1)$ & 0.66 \\
\hline Smoking status & & & & & & $<0.001$ \\
\hline Never smoker & 1568(54.8) & $774(55.8)$ & $53(37.6)$ & $623(58.0)$ & $118(45.2)$ & \\
\hline Former smoker & 247(8.6) & $118(8.5)$ & $17(12.1)$ & $84(7.8)$ & $28(10.7)$ & \\
\hline Current smoker & 1049(36.6) & $496(35.7)$ & $71(50.4)$ & $367(34.2)$ & $115(40.1)$ & \\
\hline \multicolumn{7}{|l|}{ Comorbidities } \\
\hline COPD & $12(0.4)$ & $8(0.6)$ & $1(0.7)$ & $2(0.2)$ & $1(0.4)$ & 0.47 \\
\hline Hepatic cirrhosis & $7(0.2)$ & $1(0.1)$ & $0(0.0)$ & $5(0.5)$ & $1(0.4)$ & 0.22 \\
\hline Peptic ulcer or previous GIB & $80(2.8)$ & $43(3.1)$ & $6(4.3)$ & $23(2.1)$ & $8(3.1)$ & 0.34 \\
\hline Arthritis & $46(1.6)$ & $16(1.2)$ & $3(2.1)$ & $26(2.4)$ & $1(0.4)$ & 0.03 \\
\hline Dementia & $19(0.7)$ & $8(0.6)$ & $1(0.7)$ & $9(0.8)$ & $1(0.4)$ & 0.81 \\
\hline Cancer & $48(1.7)$ & $23(1.7)$ & $5(3.6)$ & $17(1.6)$ & $3(1.2)$ & 0.32 \\
\hline Peripheral angiopathy & $21(0.7)$ & $9(0.7)$ & $1(0.7)$ & $7(0.7)$ & $4(1.5)$ & 0.47 \\
\hline Index event & & & & & & 0.01 \\
\hline Transient ischemic attack & 208(7.3) & 106(7.6) & $19(13.5)$ & $62(5.8)$ & $21(8.1)$ & \\
\hline Acute ischemic stroke & $2656(92.7)$ & $1282(92.4)$ & $122(86.5)$ & 1012(94.2)) & $240(92.0)$ & \\
\hline SBP at admission, mm Hg (median, IQR) & 150(135-167) & 150(136-165) & 150(130-165) & 150(135-170) & 150(135-162) & 0.31 \\
\hline DBP at admission, mm Hg (median, IQR) & $88(80-95)$ & 89(80-96) & $85(80-90)$ & $87(80-95)$ & $85(80-95)$ & 0.23 \\
\hline NIHSS at admission (median, IQR) & $4(1-7)$ & $3(1-5)$ & $3(1-5)$ & $4(2-8)$ & $5(2-10)$ & $<0.001$ \\
\hline Fasting blood glucose, mmol/L (median, IQR) & $5.4(4.8-6.8)$ & $5.3(4.7-6.6)$ & $5.3(4.8-6.2)$ & $5.7(4.9-7.2)$ & $5.8(4.8-7.1)$ & $<0.001$ \\
\hline TG, mmol/L (median, IQR) & $1.5(1.1-2.1)$ & $1.5(1.1-2.2)$ & $1.6(1.1-2.1)$ & $1.5(1.1-2.1)$ & $1.4(1.1-2.0)$ & 0.15 \\
\hline TC, mmol/L (median, IQR) & $4.66(4.00-5.38)$ & $4.67(4.06-5.40)$ & $4.58(3.87-5.33)$ & $4.68(3.98-5.38)$ & $4.50(3.95-5.35)$ & 0.15 \\
\hline $\mathrm{HDL}$, mmol/L (median, IQR) & $1.12(0.96-1.32)$ & $1.15(0.98-1.36)$ & $1.08(0.95-1.29)$ & $1.09(0.95-1.29)$ & $1.08(0.91-1.27)$ & $<0.001$ \\
\hline LDL, mmol/L (median, IQR) & $2.82(2.27-3.45)$ & $2.83(2.26-3.43)$ & $2.74(2.28-3.40)$ & $2.84(2.28-3.47)$ & $2.82(2.25-3.49)$ & 0.91 \\
\hline
\end{tabular}

Abbreviations: ICAS indicates intracranial atherosclerotic stenosis, ECAS extracranial atherosclerotic stenosis, SD standard deviation, COPD chronic obstructive pulmonary disease, GIB gastrointestinal bleeding, SBP systolic blood pressure, DBP diastolic blood pressure, IQR interquartile range, NIHSS National Institutes of Health Stroke Scale, $T G$ triglyceride, $T C$ cholesterol, $H D L$ high density lipoprotein, $L D L$ low density lipoprotein

However, no significant association was found between time duration of smoking and the occurrence of ICAS or specific location of ICAS (intracranial ICA, MCA, ACA, PCA and BA) (Additional file 1: Table S2).

Association between extent of smoking and ECAS or ICAS Association between extent of smoking and the occurrence of ECAS or ICAS was investigated in the subgroup of patients who were with never and current smoking ( $n=2617)$. The risk of ECAS increased steadily with larger amount of cigarette smoked per day (Fig. 1b). After adjusting for all potential confounders, significant association was found between the number of cigarette smoked per day and the occurrence of ECAS. With one cigarette smoked per day increment, the risk of developing ECAS increased by $1.0 \%$ (adjusted $\mathrm{OR}=1.010 ; 95 \% \mathrm{CI}=1.001-1.020 ; P=0.03$ ). 
Table 2 Association between smoking status and ECAS and ICAS in all enrolled patients $(N=2864)$

\begin{tabular}{|c|c|c|c|c|c|c|c|c|c|}
\hline Arterial stenosis & Category & Unadjusted OR & $P$ value & Adjusted $\mathrm{OR}_{1}$ & $P$ value & Adjusted $\mathrm{OR}_{2}$ & $P$ value & Adjusted $\mathrm{OR}_{3}$ & $P$ value \\
\hline \multirow[t]{3}{*}{ ECAS } & Previous smoking vs. never & $1.82(1.27-2.61)$ & 0.001 & $1.57(1.07-2.30)$ & 0.02 & $1.49(1.01-2.09)$ & 0.04 & $1.42(0.92-2.18)$ & 0.11 \\
\hline & Current smoking vs. never & $1.76(1.41-2.20)$ & $<0.001$ & $1.82(1.39-2.38)$ & $<0.001$ & $1.74(1.33-2.27)$ & $<0.001$ & 1.47(1.09-1.99) & $<0.01$ \\
\hline & Smoking vs. never & $1.77(1.43-2.19)$ & $<0.001$ & $1.76(1.36-2.26)$ & $<0.001$ & $1.67(1.30-2.16)$ & $<0.001$ & 1.46(1.09-1.94) & $<0.01$ \\
\hline \multirow[t]{3}{*}{ ICAS } & Previous smoking vs. never & $0.93(0.71-1.21)$ & 0.58 & $0.94(0.71-1.25)$ & 0.66 & $0.91(0.69-1.22)$ & 0.54 & $1.01(0.71-1.44)$ & 0.95 \\
\hline & Current smoking vs. never & $0.95(0.81-1.11)$ & 0.51 & $1.01(0.84-1.22)$ & 0.91 & $0.99(0.82-1.20)$ & 0.93 & $0.92(0.73-1.15)$ & 0.45 \\
\hline & Smoking vs. never & $0.94(0.82-1.10)$ & 0.45 & $1.00(0.83-1.19)$ & 0.95 & $0.97(0.82-1.17)$ & 0.77 & $0.93(0.75-1.16)$ & 0.53 \\
\hline \multirow[t]{3}{*}{ Intra-ICA } & Previous smoking vs. never & $1.61(0.96-2.72)$ & 0.07 & $1.42(0.98-2.11)$ & 0.21 & $1.39(0.80-2.42)$ & 0.25 & $1.26(0.65-2.45)$ & 0.50 \\
\hline & Current smoking vs. never & $1.58(1.14-2.18)$ & 0.005 & $1.44(0.82-2.47)$ & 0.06 & $1.34(0.91-1.98)$ & 0.14 & $1.13(0.71-1.79)$ & 0.61 \\
\hline & Smoking vs. never & $1.58(1.16-2.16)$ & 0.003 & $1.43(0.99-2.07)$ & 0.05 & $1.35(0.94-1.95)$ & 0.11 & $1.15(0.74-1.79)$ & 0.52 \\
\hline \multirow[t]{3}{*}{ MCA } & Previous smoking vs. never & $1.01(0.75-1.36)$ & 0.94 & $1.01(0.74-1.37)$ & 0.97 & $0.99(0.73-1.36)$ & 0.97 & $1.05(0.72-1.53)$ & 0.79 \\
\hline & Current smoking vs. never & $1.03(0.87-1.23)$ & 0.70 & $1.02(0.83-1.25)$ & 0.86 & $1.01(0.82-1.24)$ & 0.94 & $0.89(0.70-1.14)$ & 0.36 \\
\hline & Smoking vs. never & $1.03(0.88-1.21)$ & 0.73 & $1.02(0.84-1.23)$ & 0.87 & $1.01(0.83-1.22)$ & 0.96 & $0.92(0.73-1.16)$ & 0.50 \\
\hline \multirow[t]{3}{*}{ ACA } & Previous smoking vs. never & $1.01(0.62-1.65)$ & 0.97 & $1.17(0.69-1.97)$ & 0.56 & $1.12(0.66-1.89)$ & 0.69 & $0.93(0.49-1.76)$ & 0.83 \\
\hline & Current smoking vs. never & $0.86(0.63-1.16)$ & 0.31 & $1.09(0.76-1.56)$ & 0.64 & $1.04(0.73-1.50)$ & 0.82 & $1.00(0.65-1.54)$ & 0.99 \\
\hline & Smoking vs. never & $0.89(0.67-1.17)$ & 0.39 & $1.11(0.79-1.55)$ & 0.56 & $1.06(0.75-1.49)$ & 0.74 & $0.99(0.66-1.48)$ & 0.94 \\
\hline \multirow[t]{3}{*}{ PCA } & Previous smoking vs. never & $1.16(0.84-1.60)$ & 0.37 & $1.29(0.92-1.83)$ & 0.14 & $1.24(0.88-1.76)$ & 0.22 & $1.40(0.93-2.11)$ & 0.10 \\
\hline & Current smoking vs. never & $0.71(0.58-0.88)$ & 0.001 & $0.91(0.71-1.16)$ & 0.45 & $0.88(0.68-1.13)$ & 0.32 & $0.92(0.68-1.23)$ & 0.57 \\
\hline & Smoking vs. never & $0.79(0.65-0.96)$ & 0.01 & $0.99(0.79-1.25)$ & 0.94 & $0.96(0.76-1.21)$ & 0.72 & $1.02(0.77-1.34)$ & 0.90 \\
\hline \multirow[t]{3}{*}{ BA } & Previous smoking vs. never & $0.91(0.53-1.56)$ & 0.73 & $0.90(0.51-1.60)$ & 0.73 & $0.83(0.47-1.47)$ & 0.52 & $0.79(0.40-1.53)$ & 0.48 \\
\hline & Current smoking vs. never & $0.71(0.51-1.00)$ & 0.05 & $0.81(0.55-1.20)$ & 0.30 & $0.81(0.54-1.20)$ & 0.28 & $0.88(0.56-1.40)$ & 0.59 \\
\hline & Smoking vs. never & $0.75(0.55-1.02)$ & 0.07 & $0.83(0.58-1.20)$ & 0.33 & $0.81(0.56-1.17)$ & 0.26 & $0.86(0.56-1.32)$ & 0.48 \\
\hline
\end{tabular}

Abbreviations: ICAS indicates intracranial atherosclerotic stenosis, ECAS extracranial atherosclerotic stenosis, OR odds ration, Intra-ICA intracranial internal carotid artery, MCA middle cerebral artery, $A C A$ anterior cerebral artery, $P C A$ posterior cerebral artery, $B A$ basilar artery

$\mathrm{OR}_{1}$ adjusted for demographics (age and gender). $\mathrm{OR}_{2}$ adjusted for demographics (age and gender) and stroke risk factors (diabetes mellitus, hypertension, dyslipidemia, family history of stroke, history of cerebral ischemia, history of hemorrhagic stroke, heart disease). OR ${ }_{3}$ adjusted for all potential confounders including demographics, (age and gender), stroke risk factors (diabetes mellitus, hypertension, dyslipidemia, family history of stroke, history of cerebral ischemia, history of hemorrhagic stroke, heart disease), comorbidities (COPD, hepatic cirrhosis, peptic ulcer or previous GIB, arthritis, dementia, cancer and peripheral angiopathy), admission NIHSS, admission SBP and DBP, and admission blood tests (fasting blood glucose, TG, TC, HDL and LDL)

However, no significant association was found between extent of smoking and the occurrence of ICAS or specific location of ICAS (intracranial ICA, MCA, ACA, PCA and BA) (Additional file 1: Table S3).

\section{Association between pack years of smoking and ECAS or ICAS}

Association between pack years of smoking and the occurrence of ECAS or ICAS was investigated in the subgroup of patients who were with never and current smoking ( $n=2617)$. The risk of ECAS increased steadily with larger number of pack years of smoking (Fig. 1c). After adjusting for all potential confounders, significant association was found between pack years of smoking and the occurrence of ECAS. With one pack year of smoking increment, the risk of developing ECAS increased by $0.7 \%$ (adjusted OR $=1.007 ; 95 \% \mathrm{CI}=1.002-1.012 ; P<0.01$ ). However, no significant association was found between pack years of smoking and the occurrence of ICAS or specific location of ICAS (intracranial ICA, MCA, ACA, PCA and BA) (Additional file 1: Table S4).

\section{Discussion}

In the present study, we systematically investigated the relationship between cigarette smoking and the occurrence of ECAS and ICAS. It was found that cigarette smoking was significantly associated with the occurrence of ECAS. In addition, dose-response relationship between time duration, extent and pack years of cigarette smoking and the occurrence of ECAS was identified. However, no similar association was found between cigarette smoking and the occurrence of ICAS or specific location of ICAS (intracranial ICA, MCA, ACA, PCA, and BA).

The evidence linking cigarette smoking to stroke is extremely convincing and dose-response relationship has been well established [4, 5, 12-14]. However, the mechanisms through which cigarette smoking affects stroke risk are not well understood and have been a topic of interest over the last decades. Cigarette smoking likely contributes to increased stroke risk through both shortterm effect on thrombus generation in atherosclerotic arteries and long-term effect on the development of 

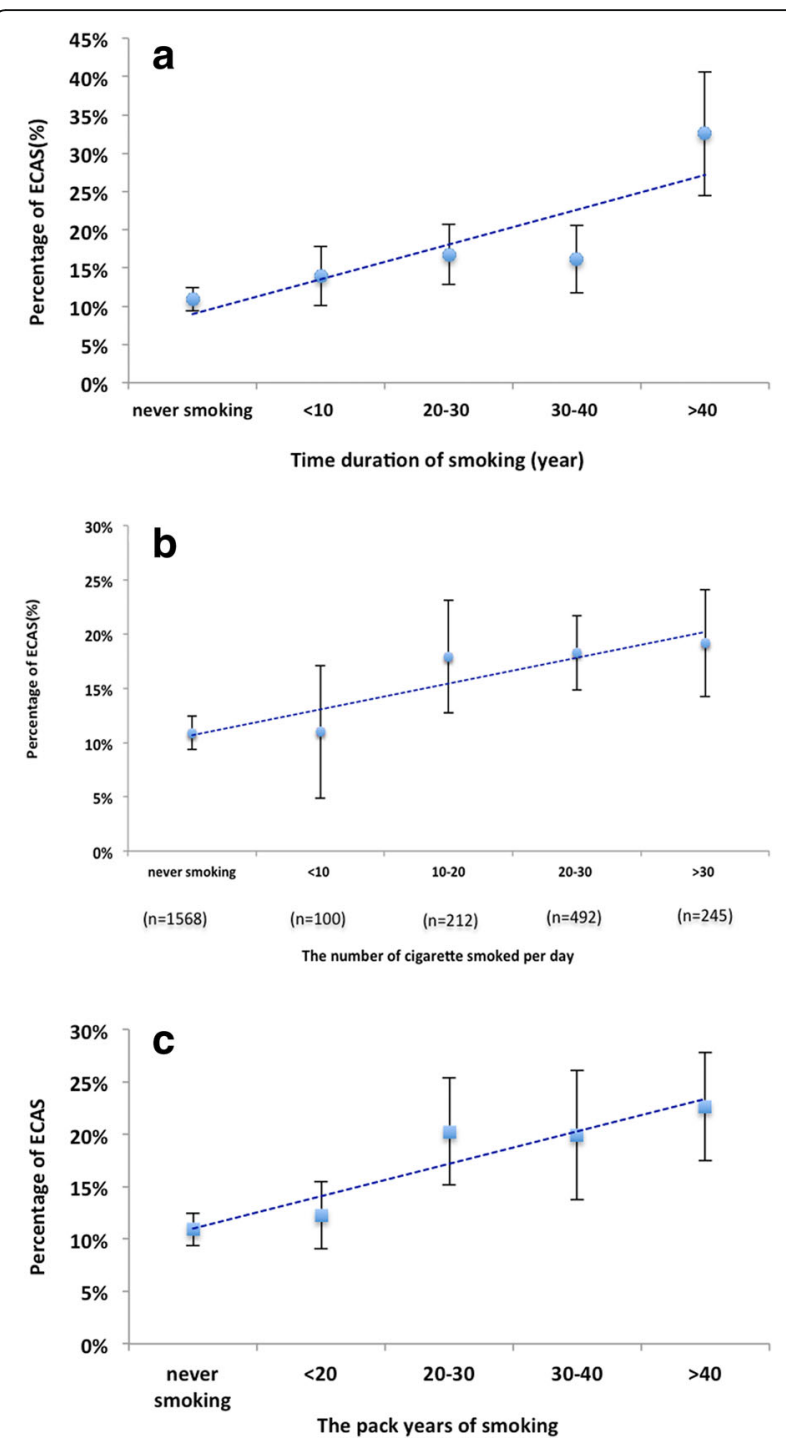

Fig. 1 relationship between cigarette smoking the development of extracranial atherosclerotic stenosis (ECAS). The risk of ECAS increased steadily with longer time duration (a), larger amount of cigarette smoked per day (b), and larger number of pack years (c) of smoking

atherosclerotic stenosis. Thus far, several studies have investigated the relationship between cigarette smoking and the occurrence of extracranial or intracranial atherosclerosis. To evaluate the prevalence and risk factors of asymptomatic carotid artery disease, Dr. Willeit and Kiechl analyzed a sample drawn from the communitybased Study and found that pack years of smoking was the leading risk factor of carotid atherosclerosis in men [15]. In a cross-sectional study carried out in patients attending a lipid clinic, Dr. Baldassarre and his colleagues found that carotid intima-media thickness was significantly associated with pack years of smoking in both former and current smokers [14]. In the Atherosclerosis Risk in Communities (ARIC) Study, Dr. Howard and his colleagues found that exposure to cigarette smoking was associated with progression of atherosclerosis. Relative to never smokers, current cigarette smoking was associated with $50 \%$ increase and past smoking was associated with $25 \%$ increase in intimal-medial thickness of carotid artery over 3 years follow-up [16]. By using transcranial Doppler ultrasonography in patients who had at least one vascular risk factor of hypertension, diabetes, or hyperlipidemia, Dr. Wong and his colleagues found that age, hypertension, diabetes, and hyperlipidemia were associated with the occurrence of MCA stenosis; however, cigarette smoking was not significant [17]. Based on data from a consecutive series of subjects aged more than 40 years and without history of stroke, Dr. Bae and his colleagues found that age, hypertension, and diabetes were significantly associated with the occurrence of ICAS; however, no significant association was observed with regard to male gender, cigarette smoking and hyperlipidemia [18]. In a meta analysis including 15 Asian studies, Dr. Ding and his colleagues found that female or patients with metabolic syndrome were more likely to suffer from ICAS than ECAS; whereas, the smoker or patients with dyslipidemia were more likely to suffer from ECAS than ICAS [19]. Similar with these findings, our study verified that cigarette smoking was more associated with ECAS than ICAS. In addition, our study provided further evidence to indicate a dose-response relationship between cigarette smoking and the occurrence of ECAS. It was shown that with 1 year of smoking, one cigarette smoked per day, and one pack year of smoking increment, the risk of developing ECAS increased by $1.0,1.1$ and $0.7 \%$, respectively. These numbers not only quantitatively showed the detrimental effect of cigarette smoking on cerebral atherosclerosis, but also suggested the potential effect of smoking cessation on stroke prevention. If we assumes an attributable risk of stroke as associated with ECAS of $0.4 \%$ per year [20] and the mean direct medical cost of ischemic stroke during acute hospitalization of $10,000 \mathrm{RMB}(\approx 1548.25$ US dollar with the exchange rate of 6.4589), taken in conjunction with the 350,000,000 smokers in China, then, if all Chinese stopped smoking, there would be 3,500,000 fewer ECAS and 14,000 fewer strokes every year. The cost benefits alone would be enormous, potentially saving $140,000,000 \mathrm{RMB}(\approx 21,675,517.50$ US dollar) per year spent on acute stroke care in China.

Although a clear association between smoking and health risk has long been established, the prevalence of smoking in our society remains alarmingly high. Smoking can be potentially reduced by population and individualrelated measures. Smoke-free laws in certain areas have already been shown to be effective in reducing heart disease [21], declining in heart disease death [22], and improving health outcomes [23]. In addition, nowadays, 
smokers have more help than ever before to quit successfully $[24,25]$. Given the clear evidence that intervention can lead to healthy change, clinicians, researchers, policymaker, social workers, smokers and their relatives should be organized together to establish reliable, feasible, and cost-effective networks and pathways to create an environment in which smoking is unacceptable and to decrease the burden of smoking on health continuously.

Indeed, limited is known about the molecular mechanisms underlying the paradoxical relationship between cigarette smoking and the development of ECAS and ICAS. Previous studies have shown that cigarette smoke exposure had complex and extensive roles in vasomotor dysfunction, inflammation, oxidative stress and modification of lipids [26-28], which are integral components for initiation and progression of atherosclerosis. Due to sorts of difference in anatomical structure and local hemodynamics, these intermediate factors induced by cigarette smoking might have different role in the development of intracranial and extracranial atherosclerosis. Further studies on molecular mechanisms are warranted.

Our study has limitations that deserve mention. First, like all observational studies, we cannot rule out the possibility that additional variable (unmeasured confounders, e.g. medications used before hospitalization) might have some impact on ECAS and ICAS. Second, our study included only hospitalized patients and those patients died in emergency room, treated in outpatient clinics and asymptomatic patients were not included. In addition, our study required informed consent and selection bias was inevitable [29]. Third, in our study, intracranial artery stenosis was evaluated with 3-D time-of-flight MRA. Currently, digital subtract angiography (DSA) remains the gold standard for the diagnosis of arterial stenosis. Although MRA is noninvasive and more easily accessible compared with DSA [30], time-of-flight MRA is prone to artifacts because of flow abnormalities [31]. Finally, information on environmental tobacco smoke was not collected. Evidence has shown that exposure to environmental tobacco smoke is an established risk factor for heart disease [32, 33]. Meanwhile, we did not collect relevant information on the type of cigarette smoked by the subjects. With these kinds of information included in the study, we would have more convincing conclusion.

\section{Conclusion}

We found a paradoxical dose-response relationship between cigarette smoking and the occurrence of ECAS and ICAS. Our study would encourage further studies to clarify molecular mechanisms underlying the different role of cigarette smoking in the development of ECAS and ICAS, as hopefully would pave ways to develop different acute care and preventive strategies for stroke patients with different smoking status.

\section{Additional file}

Additional file 1: Table S1. Proportion of intracranial and extracranial atherosclerotic stenosis. Showed the proportion of intracranial and extracranial atherosclerotic stenosis ( $n=2864$ ). Table S2. Association between time duration of smoking and ECAS and ICAS in subgroup of patients with never and current smoking. Showed the association between time duration of smoking and ECAS and ICAS in subgroup of patients with never and current smoking ( $n=2617$ ). Table S3. Association between extent of smoking and ECAS and ICAS in subgroup of patients with never and current smoking. Showed the Association between extent of smoking and ECAS and ICAS in subgroup of patients with never and current smoking ( $n=2617)$. (DOCX $46 \mathrm{~kb}$ )

\section{Abbreviations}

ACA: Anterior cerebral artery; AIS: Acute ischemic stroke; BA: Basilar artery; CICAS study: the Chinese intracranial atherosclerosis (CICAS) study; COPD: Chronic obstructive pulmonary disease; CRF: Case Report Form; CT: Computerized tomography; DBP: Diastolic blood pressure; DSA: Digital subtract angiography; DWI: Diffusion-weighted imaging; ECAS: Extracranial atherosclerotic stenosis; FLAIR: Fluid-attenuated inversion recovery sequences; GIB: Gastrointestinal bleeding; HDL: High density lipoprotein; ICA: Internal carotid artery; ICAS: Intracranial atherosclerotic stenosis; IQR: Interquartile range; IRB: Institutional Review Board; LDL: Low density lipoprotein; MCA: Middle cerebral artery; MRI: Magnetic resonance imaging; mRS: modified Rankin Scale; NIHSS: National Institutes of Health Stroke Scale; PCA: Posterior cerebral artery; SBP: Systolic blood pressure; SD: Standard deviation; TC: Cholesterol; TG: Triglyceride; TIA: Transient ischemic attack

\section{Funding}

This study was funded by the Ministry of Science and Technology and the Ministry of Health of the People's Republic of China, National S\&T Major Project of China (2008ZX09312-008), State Key Development Program of Basic Research of China (2009CB521905), and partially supported by the Nova Program of Beijing Science and Technology Commission (Dr. Ji, 2008B30), National Natural Science Foundation of China (Dr. Ji, 81,471,208, 81,641,162) and Beijing high-level healthy human resource project (215 Project) (Dr. Ji, 2014-3- 033).

\section{Availability of data and materials}

The datasets used and/or analyzed during the current study are available from the corresponding author on reasonable request.

\section{Authors' contributions}

RJ and YW (Yongjun Wang) conceived of the study, participated in its design, and drafted the manuscript. HY and YP carried out statistical analysis. RJ, YP, HY, RH, GL, PW, YW (Yilong Wang), HL, XZ, YW (Yongjun Wang) participated in analysis or interpretation of data, and revised the manuscript for important intellectual content. All authors read and approved the final manuscript.

\section{Competing interests}

The authors declare that they have no competing interests.

\section{Consent for publication}

Not applicable.

\section{Ethics approval and consent to participate}

This study was based on the Chinese intracranial atherosclerosis (CICAS) study, which had been approved by the institutional review board (IRB) of all participating hospital (IRB at Beijing Tiantan Hospital of Capital Medical University, Beijing; IRB at Beijing Tongren Hospital of Capital Medical University, Beijing; IRB at Shanghai Sixth People's Hospital, Shanghai; IRB at Tianjin Huanhu Hosptial, Tianjin; IRB at Shanxi Provincial People's Hospital, Xi'an, Shanxi; IRB at The First Affiliated Hospital of Xiamen University, Xiamen, Fujian; IRB at Xiangya Hospital Central South University, Changsha, Hunan; IRB at No. 3 People's Hospital of Chengdu, Chengdu, Sichuan; IRB at The First Affiliated Hospital of Jinan University (Guangzhou Overseas Hospital), Guangzhou, Guangdong; IRB at Handan Central Hospital, Handan, Heibei; IRB at Handan First People's Hospital, Handan, Heibei; IRB at Qingdao Municipal Hospital, Qingdao, Shandong; IRB at The First Affiliated Hospital of Zhengzhou University, Zhengzhou, Henan; IRB at The First Affiliated Hospital of Wenzhou Medical College (the First Provincial Wenzhou Hospital of Zhejiang), Wenzhou, Zhejiang; IRB at 
The Affiliated Kailuan Hospital, North China Coal Medical College, Tangshan, Hebei; IRB at The First Affiliated Hospital of Beifang Medical College, Zhangjiakou, Hebei; and IRB at Shijiazhuang Center Hospital, Shijiazhuang, Hebei). All participants provided written informed consent.

\section{Publisher's Note}

Springer Nature remains neutral with regard to jurisdictional claims in published maps and institutional affiliations.

\section{Author details}

'Department of Neurology, Tiantan Comprehensive Stroke Center, Tiantan Hospital, Capital Medical University, Beijing, China. ${ }^{2}$ China National Clinical Research Center for Neurological Diseases, Beijing, China. ${ }^{3}$ Center of Stroke, Beijing Institute for Brain Disorders, Beijing, China. ${ }^{4}$ Beijing Key Laboratory of Translational Medicine for Cerebrovascular Disease, Beijing, China. ${ }^{5}$ Beijing Key Laboratory of Brain Function Reconstruction, Beijing, China. ${ }^{6}$ Tiantan Comprehensive Stroke Center, Beijing Tiantan Hospital, Capital Medical University, No.6 Tiantanxili, Dongcheng District, Beijing 100050, China.

\section{Received: 30 November 2016 Accepted: 6 May 2017}

\section{Published online: 26 June 2017}

\section{References}

1. Schroeder SA. New evidence that cigarette smoking remains the most important health hazard. N Engl J Med. 2013:368:389-90.

2. Jha P, Ramasundarahettige C, Landsman V, Rostron B, Thun M, Anderson RN, et al. 21st-century hazards of smoking and benefits of cessation in the united states. N Engl J Med. 2013;368:341-50.

3. Thun MJ, Carter BD, Feskanich D, Freedman ND, Prentice R, Lopez AD, et al. 50-year trends in smoking-related mortality in the united states. N Engl J Med. 2013;368:351-64.

4. Meschia JF, Bushnell C, Boden-Albala B, Braun LT, Bravata DM, Chaturvedi S, et al. Guidelines for the primary prevention of stroke: a statement for healthcare professionals from the american heart association/american stroke association. Stroke. 2014;45:3754-832.

5. Kernan WN, Ovbiagele B, Black HR, Bravata DM, Chimowitz MI, Ezekowitz $M D$, et al. Guidelines for the prevention of stroke in patients with stroke and transient ischemic attack: a guideline for healthcare professionals from the american heart association/american stroke association. Stroke. 2014;45: 2160-236.

6. Kaplan RC, Tirschwell DL, Longstreth WT Jr, Manolio TA, Heckbert SR, Lefkowitz $D$, et al. Vascular events, mortality, and preventive therapy following ischemic stroke in the elderly. Neurology. 2005:65:835-42.

7. Burns DM. Epidemiology of smoking-induced cardiovascular disease. Prog Cardiovasc Dis. 2003:46:11-29.

8. Gorelick PB, Wong KS, Bae HJ, Pandey DK. Large artery intracranial occlusive disease: a large worldwide burden but a relatively neglected frontier. Stroke. 2008;39:2396-9

9. Wang $Y$, Zhao X, Liu L, Soo YO, Pu Y, Pan Y, et al. Prevalence and outcomes of symptomatic intracranial large artery stenoses and occlusions in china: the chinese intracranial atherosclerosis (cicas) study. Stroke. 2014:45:663-9.

10. Samuels OB, Joseph GJ, Lynn MJ, Smith HA, Chimowitz MI. A standardized method for measuring intracranial arterial stenosis. AJNR Am J Neuroradiol. 2000;21:643-6.

11. Grant EG, Benson CB, Moneta GL, Alexandrov AV, Baker JD, Bluth El, et al. Carotid artery stenosis: grayscale and doppler ultrasound diagnosis-society of radiologists in ultrasound consensus conference. Ultrasound Q. 2003;19: $190-8$.

12. Shah RS, Cole JW. Smoking and stroke: the more you smoke the more you stroke. Expert Rev Cardiovasc Ther. 2010;8:917-32.

13. Bhat VM, Cole JW, Sorkin JD, Wozniak MA, Malarcher AM, Giles WH, et al. Dose-response relationship between cigarette smoking and risk of ischemic stroke in young women. Stroke. 2008;39:2439-43.

14. Baldassarre D, Castelnuovo S, Frigerio B, Amato M, Werba JP, De Jong A, et al. Effects of timing and extent of smoking, type of cigarettes, and concomitant risk factors on the association between smoking and subclinical atherosclerosis. Stroke. 2009:40:1991-8.

15. Willeit J, Kiechl S. Prevalence and risk factors of asymptomatic extracranial carotid artery atherosclerosis. A population-based study. Arterioscler Thromb. 1993;13:661-8
16. Howard G, Wagenknecht LE, Burke GL, Diez-Roux A, Evans GW, McGovern P, et al. Cigarette smoking and progression of atherosclerosis: the atherosclerosis risk in communities (aric) study. JAMA. 1998;279:119-24.

17. Wong KS, Ng PW, Tang A, Liu R, Yeung V, Tomlinson B. Prevalence of asymptomatic intracranial atherosclerosis in high-risk patients. Neurology. 2007;68:2035-8

18. Bae HJ, Lee J, Park JM, Kwon O, Koo JS, Kim BK, et al. Risk factors of intracranial cerebral atherosclerosis among asymptomatics. Cerebrovasc Dis. 2007;24:355-60.

19. Ding X, Li C, Yu K, Gao A, Xiao L, Peng F, et al. Different risk factors between intracranial and extracranial atherosclerotic stenosis in asian population: a systematic review and meta-analysis. Int. J. Neurosci. 2014;124:834-40.

20. den Hartog AG, Achterberg S, Moll FL, Kappelle LJ, Visseren FL, van der Graaf Y, et al. Asymptomatic carotid artery stenosis and the risk of ischemic stroke according to subtype in patients with clinical manifest arterial disease. Stroke. 2013:44:1002-7.

21. Lin $H$, Wang $H$, Wu W, Lang $L$, Wang $Q$, Tian $L$. The effects of smoke-free legislation on acute myocardial infarction: a systematic review and metaanalysis. BMC Public Health. 2013;13:529.

22. Fichtenberg CM, Glantz SA. Association of the california tobacco control program with declines in cigarette consumption and mortality from heart disease. N Engl J Med. 2000;343:1772-7.

23. Song AV, Dutra LM, Neilands TB, Glantz SA. Association of smoke-free laws with lower percentages of new and current smokers among adolescents and young adults: an 11-year longitudinal study. JAMA Pediatr. 2015;169: e152285.

24. Rigotti NA, Clair C, Munafo MR, Stead LF. Interventions for smoking cessation in hospitalised patients. Cochrane Database Syst Rev. 2012;16(5):CD001837.

25. Stead LF, Lancaster T. Combined pharmacotherapy and behavioural interventions for smoking cessation. Cochrane Database Syst. Rev. 2012;10: CD008286.

26. Arnson $Y$, Shoenfeld $Y$, Amital $H$. Effects of tobacco smoke on immunity, inflammation and autoimmunity. J Autoimmun. 2010;34:J258-65.

27. Weber $\mathrm{C}$, Noels $\mathrm{H}$. Atherosclerosis: current pathogenesis and therapeutic options. Nat Med. 2011;17:1410-22

28. Starke RM, Ali MS, Jabbour PM, Tjoumakaris SI, Gonzalez F, Hasan DM, et al. Cigarette smoke modulates vascular smooth muscle phenotype: implications for carotid and cerebrovascular disease. PLoS One. 2013:8:e71954

29. Tu JV, Willison DJ, Silver FL, Fang J, Richards JA, Laupacis A, et al. Impracticability of informed consent in the registry of the canadian stroke network. $N$ Engl J Med. 2004;350:1414-21.

30. Man BL, Fu YP, Chan YY, Lam W, Hui CF, Leung WH, et al. Use of magnetic resonance angiography to predict long-term outcomes of ischemic stroke patients with concurrent stenoses in hong kong. Cerebrovasc Dis. 2009;28:112-8.

31. Nederkoorn PJ, Elgersma OE, Mali WP, Eikelboom BC, Kappelle LJ, van der Graaf $Y$. Overestimation of carotid artery stenosis with magnetic resonance angiography compared with digital subtraction angiography. J Vasc Surg. 2002;36:806-13.

32. Barnoya J, Glantz SA. Cardiovascular effects of secondhand smoke: nearly as large as smoking. Circulation. 2005;111:2684-98.

33. Awawdi K, Steiner H, Green MS, Zelber-Sagi S. Association between secondhand smoking and acute coronary heart disease among arab women with multiple risk factors. Eur J Pub Health. 2016;26:141-5.

\section{Submit your next manuscript to BioMed Central and we will help you at every step:}

- We accept pre-submission inquiries

- Our selector tool helps you to find the most relevant journal

- We provide round the clock customer support

- Convenient online submission

- Thorough peer review

- Inclusion in PubMed and all major indexing services

- Maximum visibility for your research

Submit your manuscript at www.biomedcentral.com/submit
C) Biomed Central 